\title{
Pseudotumor Deltoideus Case Report: An Incidental Finding After a Proximal Humerus Fracture
}

\section{Sager Hanna*, Bader Al-Hindi and Aliaa Khaja}

Orthopedic Surgeon (Resident), Al Razi Orthopedic Hospital, Kuwait Institute for Medical Specialization, Kuwait

*Corresponding Author: Sager Hanna, Orthopedic Surgeon (Resident), Al Razi Orthopedic Hospital, Kuwait Institute for Medical Specialization, Kuwait City, Kuwait.
Received: March 16, 2020

Published: March 30, 2020

(C) All rights are reserved by Sager Hanna., et al.

\begin{abstract}
Introduction: Morgan., et al. first reported pseudotumor deltoideus in 2001 in a study of five cases of chronic or subacute, painful and asymptomatic pseudotumor deltoideus in the deltoid insertion. This peculiar diagnosis should be considered when evaluating cortical abnormalities in the area of the deltoid insertion.

Case Presentation: This paper presents the incidental radiological findings of a 39-year old male of Arabic background, with acute left shoulder pain after trauma. Initial radiographs of the left shoulder showed a fracture of the left proximal humerus, as well as a uni-lamellated periosteal reaction on the lateral cortex of the humerus at the level of the deltoid tuberosity.

Conclusion: The final diagnosis of pseudotumor deltoideus was made based on clinical as well as radiologic findings. Correctly identifying and treating these latent cases with conservative measure is critical to orthopedic surgeons. However, further studies are needed to assess long term follow up and whether watchful waiting and observation is the best method of care in such cases.
\end{abstract}

Keywords: Pseudotumor Deltoideus; Proximal Humerus Fracture; Deltoid Insertion

\section{Introduction}

Morgan., et al. first reported pseudotumor deltoideus in 2001 in a study of five cases of chronic or subacute, painful, and asymptomatic pseudotumor deltoideus in the deltoid insertion. This peculiar diagnosis should be considered when evaluating cortical abnormalities in the area of the deltoid insertion. Benign skeletal anatomic variants associated with disability and pain are well-established entities that may simulate infections or neoplasms [1]. Intracortical desmoid, or avulsive cortical irregularity is one such variant [1]. Another are the herniation pits of the anterior femoral neck $[2,3]$. The combination of radiographic changes and pain in these regions has occasionally prompted biopsy as clinicians who are unfamiliar with the morphology of these lesions may suspect malignancy.

An anatomic variant in the proximal humerus of the left shoulder at the site of deltoid insertion was observed in a 39-year old previously healthy subject. This report is a presentation of the case, describing the imaging and clinical features of this variant known as pseudotumor deltoideus.

\section{Case Presentation}

A 39-year old previous healthy man presented to the casualty department complaining of left shoulder swelling, pain, and inability to move his shoulder after a simple fall from standing height onto his left shoulder. He is previously healthy and right-hand dominant. There was no history of loss of consciousness, head injury or focal neurological deficit.
On physical examination of his left shoulder he had swelling, restricted range of motion, and tenderness over the shoulder joint and proximal humerus. His distal neuro-vascular status was intact.

Initial radiographs of the left shoulder (Figure 1) showed a fracture of the left proximal humerus, as well as a uni-lamellated periosteal reaction on the lateral cortex of the humerus at the level of the deltoid tuberosity.

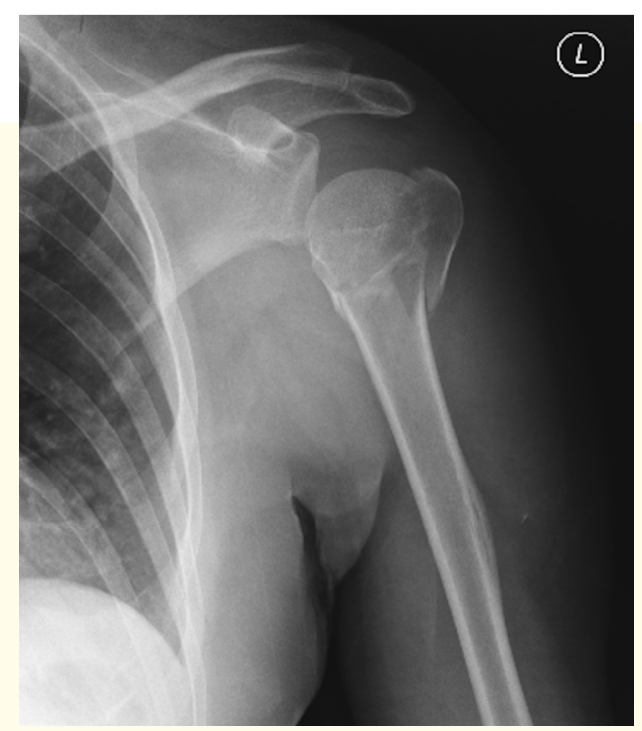

Figure 1: Initial anterior-posterior radiograph of the left shoulder. The radiograph shows a proximal humerus fracture and a uni-lamellated periosteal reaction of the lateral cortex of the humerus, at the level of the deltoid insertion. 
As the treatment plan for the patient was surgical fixation, a computed tomography (CT) scan of the proximal humerus was ordered for pre-operative assessment of fracture fragments (Figure 1). The CT scan revealed fracture through the greater tuberosity and surgical neck, but no intra-articular extension. Lateral cortex of the humerus was seen to have a thickened layer of periosteal reaction with a radiolucent center (Figure 2). The axial view further demonstrated this lesion (Figure 3). Routine laboratory investigations including complete blood count, serum electrolytes and a coagulation profile showed no abnormalities. Erythrocyte sedimentation rate, as well as c-reactive protein were ordered. These two investigations turned up normal.

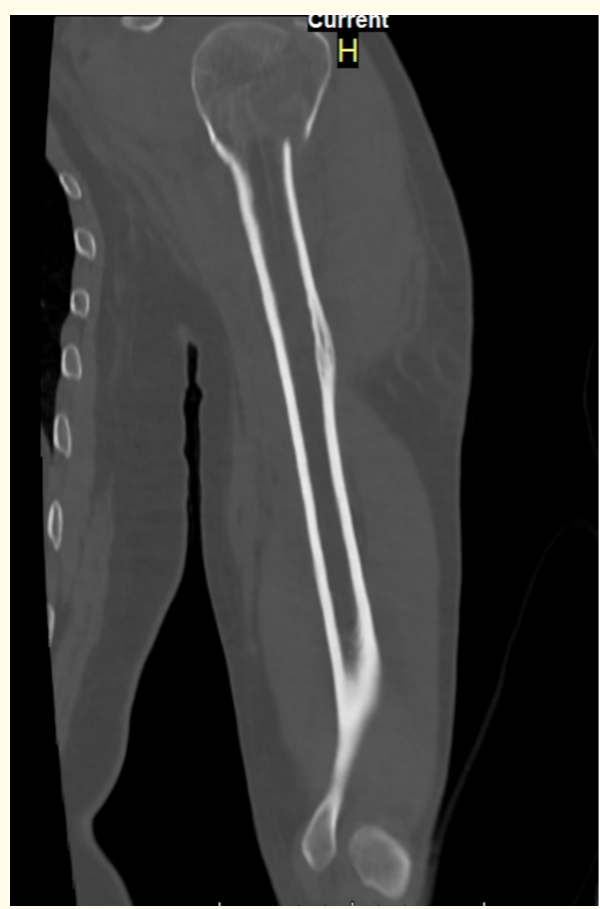

Figure 2: Coronal view CT scan of the left shoulder. The CT scan reveals the fracture pattern as well as the lesion at the level of the deltoid insertion.

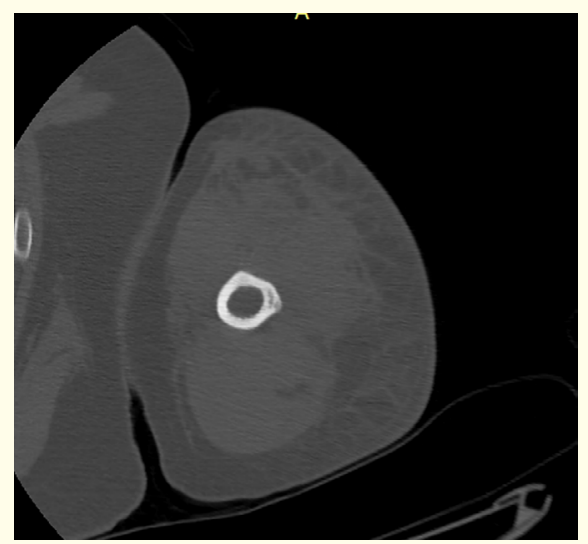

Figure 3: Axial view CT scan of the left humerus. The axial cut further delineates the thickened lateral cortex and radiolucent center of the lesion.
After correlation with clinical and laboratory findings, the radiological findings were considered benign and the patient was asymptomatic before the trauma. Therefore, A magnetic resonance imaging was not obtained. Operative treatment of the fracture with open reduction and internal fixation with proximal humerus locking plate and screws was undertaken for the patient (Figure 4). He was kept four days postoperatively then discharged for follow up in the outpatient department. He is currently undergoing regular follow ups and physiotherapy program to restore the range of motion and strength of his left shoulder.

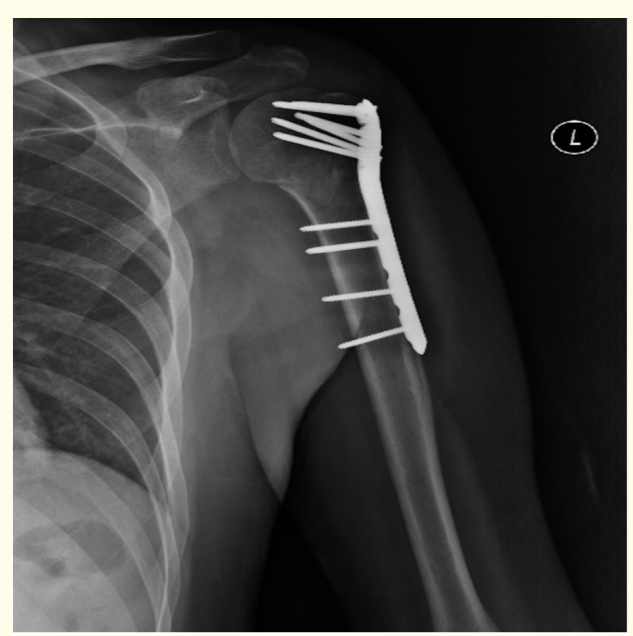

Figure 4: Post-operative anterior-posterior radiograph of the left shoulder. Post-operative radiograph of the left shoulder shows the fracture being treated with open reduction and internal fixations using plate and screws. Meanwhile, the deltoideus pseudotumor was left for watchful waiting and follow up.

\section{Discussion}

Incidental findings of lesions in the axial and non-axial skeleton is a common reason for referral to the orthopedic clinic. When the bony irregularity or lucency is associated with tenderness, pain, or a high uptake on bone scan, then radiographic results may suggest a neoplastic process [1]. Other anatomic variants which may be analogous in their etiology include accentuation of the normal deltoid insertional anatomy, herniation pits, and avulsive cortical irregularities.

We do not have a full understanding of the exact etiology of pseudotumor deltoideus. Given the wide spectrum of imaging studies, pseudotumor deltoideus might have several explanations. Potential explanations for pseudotumor deltoideus may include burned out or indolent neoplasms, erosions slinked with tendonitis, and other similar anatomic variants.

Herniation pits may appear to be closely linked to the lucencies seen in our case study. Herniation pits are fibrous pits that occur at the joint capsule insertion on the anterolateral femoral neck [2,3]. Plain radiographs illustrates herniation pits as rounded, variably 
sized lucencies. While herniation pits may be considered a normal variant, they may have some associated symptoms, as was found by Daenen., et al. [2] Similarities shared with the cystic form of pseudotumor deltoideus include the cystic nature of either bone abnormalities, the unique location at the site of insertion, and the fact that they can be linked with symptoms despite notwithstanding their benign radiographic appearance.

Similar clinical and radiographic findings to those with the cortical variant of pseudotumor deltoideus could be observed in patients having distal femur avulsive cortical irregularities (ACI), also referred to as intracortical desmoid [4-6]. ACI has a radiographic appearance of radiolucent cortical irregularities or defects on the posteromedial region of the distal femoral metaphysis. It is important to note that some unique differences exist between pseudotumor deltoideus, and ACI apart from their distinct locations. ACI lesions occur mostly in children bearing exposed physes. However, our patient with pseudotumor deltoideus was skeletally mature.

Bone erosions in calcific tendinitis that bear semblance to those seen in pseudotumor deltoideus have been described in a particular case $[6,7]$. Such cases are characterized by calcium deposits in the adjacent soft tissues, on either computed tomography or plain radiographs.

While the chances of a pseudotumor deltoideus representing a tumor is slim, two benign entities that bear similarities to neoplasm, receive special consideration. Fibrous cortical defects and non-ossifying fibromas are indolent, eccentric lesions $[7,8]$. But that said, they differ from pseudotumor deltoideus in that they occur mostly between four and eight years of age, rarely occur postpuberty, are metaphyseal, and rarely manifests symptoms where there is no pathologic fracture [7-9]. They also resolve over time. Solitary fibrous dysplasia occurs in long bone diaphysis. It is a benign fibrous bone tumor that presents in association with pain or observed incidentally and is associated with high uptake on bone scan [8]. However, the radiographic properties of fibrous dysplasia, such as the central location, cortical thinning, and excessive bulbous expansion of the bone differ from those of the entity currently described [8-10].

Neoplasms and other pain sources should be excluded during evaluation of bone abnormalities that are thought to be pseudotumor deltoideus.

Infection was ruled out by the patient's history as there was no history of fever, recent contact with susceptible individuals, and no history of localized pain shoulder or around the arm. In addition, laboratory investigations were normal, and no bone/joint destruction as evident on radiological imaging.

The final diagnosis of pseudotumor deltoideus was made based on clinical and radiological findings, especially after excluding the aforementioned differentials.

\section{Conclusion}

Lucency or bony irregularity at the deltoid insertion on the proximal end of the humerus is an uncommon radiographic finding. Yet, many orthopedic surgeons will be presented with patients who suffer from shoulder pain. This case report demonstrates the different diagnoses that could potentially be associated with such pain, and sheds light on the slight differences that exist between them. The incidental finding was determined to be a deltoideus pseudotumor based on not only the radiographic features, but history and examination as well as laboratory studies. Emphasis should be made on long term follow up, and further studies are needed to evaluate this method of treating patients with this condition.

\section{Consent}

The authors declare that informed consent has been obtained from the patient.

\section{Competing Interests}

The authors declare that they have no competing interests.

\section{Authors' Contributions}

SH had the task of doing a literature review of similar cases that have relevance to our report. He revised the final draft of the manuscript and collected the images used in the report. He also helped in going over the references used in this report.

BA was the treating surgeon; he was in charge of initial casualty assessment as well as operative management and the patient follows up in his clinic. He presented this case as a case of interest for the report.

AK prepared the study design as well as writing the first draft and reviewing the manuscript. She was also responsible for keeping the team on schedule with respect to timeframe available and allocation of roles.

All co-authors reviewed and accepted the final draft of the manuscript as well as the corresponding figures.

\section{Bibliography}

1. Morgan H., et al. "Pseudotumor deltoideus: a previously undescribed anatomic variant at the deltoid insertion site". Skeletal Radiology 30.9 (2001): 512-518.

2. Adiguzel E., et al. "A Rare Cause of Shoulder Pain". American Journal of Physical Medicine and Rehabilitation 94.10 (2015): e96-e97.

3. Aykol G and Elbir S. "Pseudotumor Deltoideus in the Left Humerus of a Young Adult Female Patient with Acute Lateral Shoulder Pain: A Case Report". Medicine Science / International Medical Journal 5.1 (2016): 330.

4. Fulton MN., et al. "Cortical desmoid-like lesion of the proximal humerus and its occurrence in gymnasts (ringman's shoulder lesion)". American Journal of Sports Medicine 7.1 (1979): 57 61. 
5. Yamazaki T., et al. "MR findings of avulsive cortical irregularity of the distal femur". Skeletal Radiology 24.1 (1995): 43-46.

6. Fritz P., et al. "Paradiaphyseal calcific tendinitis with cortical bone erosion". Arthritis and Rheumatology 37.5 (1994): 718723.

7. Huvos AG. "Nonossifying fibroma, benign fibrous histiocytoma, and xanthoma of bone". In: Huvos AG, ed. Bone tumors: diagnosis, treatment, and prognosis. $2^{\text {nd }}$ edition. Philadelphia: WB Saunders (1991): 481-496.

8. Huvos AG. "Ossifying fibroma and fibrous dysplasia”. In: Huvos $\mathrm{AG}$, ed. Bone tumors: diagnosis, treatment, and prognosis. $2^{\text {nd }}$ edition. Philadelphia: WB Saunders (1991): 13-48.

9. Anderson SE., et al. "Latissimus dorsi tendinosis and tear: imaging features of a pseudotumor of the upper limb in five patients". American Journal of Roentgenology 185.5 (2005): 1145-1151.

10. Damron TA., et al. "Diagnosis and treatment of joint related tumors that mimic sports-related injuries". Instructional Course Lectures 58 (2009): 833-847.

\section{Assets from publication with us}

- Prompt Acknowledgement after receiving the article

- Thorough Double blinded peer review

- Rapid Publication

- Issue of Publication Certificate

- High visibility of your Published work

Website: https://www.actascientific.com/

Submit Article: https://www.actascientific.com/submission.php Email us: editor@actascientific.com

Contact us: +919182824667 